\title{
极小子流形的曲率估计与稳定性*
}

\author{
沈 - 兵 \\ (杭州大学数学系)
}

摘 要

本文给出空间形式中极小子流形共形度量的曲率上界估计，并用来研究极小子 流形的稳定性. 这就部分地解答了下述问题：已给极小子流形 $M^{n} \hookrightarrow \widetilde{M}^{n+p}$ ，寻 找一个仅与 $M^{n}$ 和 $\widetilde{M}^{n+p}$ 的度量有关的条件, 使得若区域 $\mathscr{D} \subset M^{n}$ 满足这个条件, 则 $\mathscr{D}$ 是稳定的.

设 $M^{2}$ 是 $\mathrm{R}^{3}$ 中非全测地的极小曲面, 对于 $M^{2}$ 上诱导度量 $g$, 古典的 Ricci 条件是, 共 形度量 $\tilde{g}=-K g$ 具有常数 Gauss 曲率 $\widetilde{K}=1$, 这里 $K$ 是关于 $g$ 的 Gauss 曲率. BarbosaDo Carmo ${ }^{[1]}$ 和 Chern-Osserman ${ }^{[2]}$ 已作出了高维极小超曲面上的各种推广. 特别是单连通空间 形式中的极小曲面，共形度量的 Gauss 曲率 $\widetilde{K}$ 的上界估计与极小曲面的稳定性密切相关 ${ }^{[3]}$. 有关极小子流形的稳定性，Barbosa-DoCarmo ${ }^{[4,5]}$, Mori ${ }^{[6]}$,Spruck ${ }^{[7]}$ 等已有不少结果。

在本文中,我们要给出实空间形式中一般极小子流形共形度量的纯量曲率的上界估计(见 第二节, 定理 1), 并把它用来研究极小子流形的稳定性, 从而推广了文献 [3] 的结果 (见第三 节, 定理 2). 假设 Riemann 流形 $\widetilde{M}^{n+p}$ 中已给 $n$ 维极小子流形 $M^{n}$, 寻找一个仅与 $M^{n}$ 和 $\widetilde{M}^{n+p}$ 的度量有关的简便条件，使得当区域 $\mathscr{D} \subset M^{n}$ 满足这个条件时， $\mathscr{D}$ 是稳定的. 这是 一个饶有兴趣的问题 ${ }^{[9]}$. 就我们所知, 在 $n \geqslant 3$ 的区域稳定性方面, 至今仅有 Spruck ${ }^{[7]}$ 和 Mor i ${ }^{i 6]}$ 的工作. 本文的定理 3 和 4 试图部分地解答这个问题. 最后，作为例子，我们给出了 单位球面 $S^{n+p}(1)$ 中 Clifford 极小超曲面和Veronese 极小子流形 ${ }^{[9]}$ 上稳定测地盘的大小范 围。

\section{一、准 备工作}

我们先固定某些记号. 用 $\boldsymbol{F}^{n+p}(c)$ 表示一个具有常数截曲率 $c$ 的 $n+p$ 维实空间形 式. 设 $\left(M^{n}, g\right)$ 是 $\boldsymbol{F}^{n+p}(c)$ 中 $n(\geqslant 2)$ 维极小子流形, 其上诱导度量为 $g$. 在 $\boldsymbol{F}^{n+n}(c)$ 中选取局部正交规范标架场 $\left\{e_{A}\right\}$, 使得限制在 $M^{n}$ 上时, 向量 $\left\{e_{i}\right\}$ 与 $M^{n}$ 相切. 以下若 无其他说明，指标的取值范围约定如下:

$$
1 \leqslant A, B, C, \cdots \leqslant n+p ; \quad 1 \leqslant i, j, k, \cdots \leqslant n ; n+1 \leqslant \alpha, \beta, \cdots \leqslant n+n .
$$
设 $\left\{\omega_{A}\right\}$ 是 $\left\{e_{A}\right\}$ 的对偶标架场. 那么, $M^{n}$ 的诱导度量 $g$ 和第二基本形式 $B$ 可写 为 $^{[9]}$ : 


$$
\begin{aligned}
& g=\sum_{i} \omega_{i} \otimes \omega_{i}, \\
& B=\sum_{a, i, j} h_{i j}^{a} \omega_{i} \otimes \omega_{j} \otimes e_{a},
\end{aligned}
$$

其中 $h_{i j}^{a}=h_{i i}^{a}$ 是 $M^{n}$ 关于单位法向量 $e_{a}$ 的第二基本形式 $B_{a}$ 的系数. 极小条化就是

$$
\sum_{i} h_{i i}^{a}=0 \text { (对一切 } \alpha \text { ). }
$$

$M^{n}$ 的 Gauss-Codazzi 方程是 ${ }^{[9]}$

$$
\begin{gathered}
R_{i, k l}=c\left(\delta_{i k} \delta_{l l}-\delta_{l l} \delta_{l k}\right)+\sum_{a}\left(h_{i k}^{a} h_{l l}^{a}-h_{l l}^{a} h_{i k}^{a}\right), \\
h_{i j k}^{a}=h_{i k j}^{a},
\end{gathered}
$$

其中 $h_{i j k}^{a}$ 是 $h_{i j}^{a}$ 的广义共变导数.

由 (1.3) 和 (1.4) 式得 $\left(M^{n}, g\right)$ 的纯量曲率 $R$ 为:

$$
R=n(n-1) c-|B|^{2},
$$

其中 $|B|^{2}=\sum_{a, i, j}\left(h_{n}^{a}\right)^{2}$ 是 $B$ 的长度平方. 用 $\Delta$ 表示 $M^{n}$ 上关于 $g$ 的 Laplacian. 在文献 [8] 中已证得

$$
\frac{1}{2} \Delta\left(|B|^{3}\right) \geqslant|\nabla B|^{2}-\left(2-\frac{1}{p}\right)|B|^{4}+n c|B|^{2} \text {, }
$$

真中 $|\nabla B|^{2}=\sum_{a, \lambda, j, k}\left(h_{a, k}^{a}\right)^{2}$.

现在建立一个以后要用的命题。

命题 1. 设 $M^{n}(n \geqslant 2)$ 是 $\boldsymbol{F}^{n+n}(c)$ 的极小子流形, $B$ 是 $M^{n}$ 的第二基本形式. 那末，

$$
\left|\nabla\left(|B|^{2}\right)\right|^{2} \leqslant \frac{4 n}{n+2}|B|^{2} \cdot|\nabla B|^{2} \text {. }
$$

证. 令 $\left|B_{a}\right|^{2}=\sum_{i=1}\left(h_{i j}^{a}\right)^{2}$, 从而 $|B|^{2}=\sum_{a}\left|B_{a}\right|^{2}$. 对于任一点 $x \in M^{n}$ 和某一固定的 $\alpha$, 我们可选取局部标架场, 使得在 $x$ 有

$$
h_{i}^{a}=0 \quad(i \neq j) .
$$

于是,

$$
\begin{aligned}
\left|\nabla\left(\left|B_{a}\right|^{2}\right)\right|^{2} & =4 \sum_{k}\left(\sum_{i, b} h_{i j}^{a} h_{i, k}^{a}\right)^{2} \\
& =4 \sum_{k}\left(\sum_{i} h_{i t}^{a} h_{t, k}^{a}\right)^{2} \\
& \leqslant 4\left[\sum_{i}\left(h_{i i}^{a}\right)^{2}\right] \cdot\left[\sum_{i, k}\left(h_{i+k}^{a}\right)^{2}\right]=4\left|B_{\alpha}\right|^{2} \cdot\left[\sum_{i, k}\left(h_{t+k}^{a}\right)^{2}\right] .
\end{aligned}
$$

另一方面，以 (1.5) 式得

$$
\begin{aligned}
\sum_{i, 1, k}\left(h_{i, k}^{a}\right)^{2} & \geqslant 3 \sum_{i \neq k}\left(h_{i i k}^{a}\right)^{2}+\sum_{i}\left(h_{i i i}^{a}\right)^{2} \\
& =2 \sum_{i \neq k}\left(h_{i, k}^{a}\right)^{2}+\sum_{i, k}\left(h_{i, k}^{a}\right)^{2} .
\end{aligned}
$$


对于一个固定指标 $k$ ，根据极小性 (1.3) 式, 易见

$$
\begin{aligned}
\sum_{i}\left(h_{i i k}^{a}\right)^{2} & =\sum_{i \neq k}\left(h_{i i k}^{a}\right)^{2}+\left(\sum_{i \neq k} h_{i i k}^{a}\right)^{2} \\
& \leqslant \sum_{i \neq k}\left(h_{i+k}^{a}\right)^{2}+(n-1) \sum_{i \neq k}\left(h_{i i k}^{a}\right)^{2}=n \sum_{i \neq k}\left(h_{i, k}^{a}\right)^{2} .
\end{aligned}
$$

上式两边对 $k$ 作和，得

$$
\sum_{i \neq k}\left(h_{i i k}^{a}\right)^{2} \geqslant \frac{1}{n} \sum_{i, k}\left(h_{i i k}^{a}\right)^{2}
$$

由它和 (1.10) 式得出

$$
\sum_{i, j, k}\left(h_{i j k}^{a}\right)^{2} \geqslant \frac{n+2}{n} \sum_{i, k}\left(h_{i i k}^{a}\right)^{2} .
$$

这样, 从 (1.9) 和 (1.11) 式可得在 $x$

$$
\left|\nabla\left(\left|B_{a}\right|^{2}\right)\right|^{2} \leqslant \frac{4}{n+2}\left|B_{a}\right|^{2} \cdot\left[\sum_{i, j, k}\left(h_{i, k}^{a}\right)^{2}\right] \leqslant \frac{4}{n+2}|B|^{2} \cdot\left[\sum_{i, 1, k}\left(h_{i ; k}^{a}\right)^{2}\right],
$$

这与标架的选取无关. 因此, 在 (1.12) 式中对 $\alpha$ 作和,立即可得 (1.8) 式. 由于 $x \in M^{n}$ 是任 㽜的, 命题得证.

在结束本节之前, 对极小子流形 $M^{n} C \longrightarrow F^{n+p}(c)$ 的稳定性区域作些说明. 设 $\mathscr{D} \subset M^{n}$ 是一个区域,具有紧致闭包 $\mathscr{\mathscr { D }}$ 和逐片光滑边界 $\partial \mathscr{D}$. 极小性意味着, 对于保持 $\partial \mathscr{D}$ 不变的 $\mathscr{D}$ 的一切变分, $\mathscr{D}$ 是关于诱导度量 $g$ 的体积泛函的临界点. 设 $V=\sum_{a} v_{\alpha} e_{\alpha}$ 是 $\overline{\mathscr{D}}$ 的一 个法向量场,它在 $\partial \mathscr{D}$ 上消失, 则沿 $V$ 方向的第二变分公式是 ${ }^{[9]}$

$$
\boldsymbol{I}(V, V)=-\int_{\varnothing}\left\{\sum_{a} v_{\alpha}\left(\Delta v_{\alpha}+n c v_{\alpha}+\sum_{\beta, z, j} h_{i j}^{a} h_{i}^{\beta} v_{\beta}\right)\right\} * 1 g,
$$

其中 ${ }^{*} 1 g=\omega_{1} \wedge \cdots \wedge \omega_{n}$ 是 $\left(M^{n}, g\right)$ 的体积元.

若对于一切变分向量场 $V,\left.V\right|_{\partial \mathscr{D}}=0$, 恒有 $I(V, V) \geqslant 0$, 则称区域 $\mathscr{D} \subset M^{n}$ 是稳 定的. 一个法向量场 $V$ 被称为 Jacobi 场, 如果它满足下列椭圆线性方程组:

$$
\Delta v_{\alpha}+n c v_{\alpha}+\sum_{\beta, i, j} h_{i j}^{a} h_{i j}^{\beta} v_{\beta}=0 .
$$

关于极小子流形的 Jacobi 场和 Morse 指标定理的更完整描述，可在文献 [10]和文献 [11] 中找到。

\section{二、曲率估 计}

设 $\left(M^{n}, g\right)$ 是 $F^{n+p}(c)$ 的 $n(\geqslant 2)$ 维极小子流形. 令

$$
\sigma=n(n-1) a c-R,
$$

其中 $R$ 是关于 $g$ 的纯舅曲率, $a$ 是满足下列条件的任意实数:

$$
\left.\begin{array}{l}
a \geqslant 1+1 /\left[2(n-1)\left(2-\frac{1}{p}\right)-1\right], \quad \text { 当 } c>0, \\
a \leqslant 1-1 /\left[4(n-1)\left(2-\frac{1}{p}\right)-1\right], \quad \text { 当 } c<0 .
\end{array}\right\}
$$

把 (1.6) 式代人 (2.1) 式, 得 


$$
\sigma=|B|^{2}+n(n-1)(a-1) c .
$$

由此连同 (2.2) 式, 可见无论 $c>0$ 还是 $c<0$, 均有 $\sigma>0$. 而且, 在 $c=0$ 的情况下, 由 （2.3）式可知, 当且仅当 $|B|^{2} \neq 0$ 时， $\sigma>0$. 因此, 当 $M^{n}$ 是 $\mathbb{R}^{n+p}$ 的子流形时, 我们以后 总假定 $M^{n}$ 不是全测地的.

这样, 在 $M^{n}$ 上就可以引人共形度量 $\tilde{g}=\sigma g$. 我们要给出关于度量 $\tilde{g}$ 的纯量曲率 $\widetilde{R}$ 的上界估计, 它可看成一个 Riemann 流形 $\left(M^{n}, g\right)$ 能极小浸入 $\boldsymbol{F}^{n+p}(c)$ 的一种内蕴的必 要条件。

定理 1. 设 $\left(M^{n}, g\right)$ 是 $F^{n+p}(c)$ 的 $n(\geqslant 2)$ 维极小子流形, $R$ 是关于 $g$ 的纯量曲率. 用 $\tilde{R}$ 表示共形度量 $\tilde{g}=[n(n-1) a c-R] g$ 的纯量曲率, 其中 $a$ 是实数, 使得当 $c>0$ 时, $a \geqslant 1+1 /\left[2(n-1)\left(2-\frac{1}{p}\right)-1\right]$; 当 $c<0$ 时,

$$
a \leqslant 1-1 /\left[4(n-1)\left(2-\frac{1}{p}\right)-1\right] \text {. }
$$

那末, 必有

$$
\widetilde{R} \leqslant 2(n-1)\left(2-\frac{1}{p}\right)-1 .
$$

证. 如所知, 对于共形度量 $\tilde{g}=\sigma g$, 纯量曲率 $\widetilde{R}$ 可表示成(见文献 [12]，p. 23)

$$
\sigma \widetilde{R}=R-(n-1) \Delta \log \sigma-\frac{1}{4}(n-1)(n-2)|\nabla \log \sigma|^{2} .
$$

把(2.1) 式代入(2.5) 式,我们有

$$
-\sigma \widetilde{R}=\sigma-n(n-1) a c+(n-1) \frac{\Delta \sigma}{\sigma}+\frac{1}{4}(n-1)(n-6)|\nabla \log \sigma|^{2} .
$$

将 (1.7) 和 (2.3) 式导入 (2.6) 式, 可得

$$
\begin{gathered}
-\sigma \widetilde{R} \geqslant \sigma-n(n-1) a c-2(n-1)\left(2-\frac{1}{p}\right) \frac{|B|^{4}}{\sigma}+2 n(n-1) c \frac{|B|^{2}}{\sigma} \\
+2(n-1) \frac{|\nabla B|^{2}}{\sigma}+\frac{1}{4}(n-1)(n-6)|\nabla \log \sigma|^{3}
\end{gathered}
$$

另一方面, (2.3) 式和 (2.2) 式给出 $\sigma \geqslant|B|^{2}$. 根据命题 1, 从 (1.8) 式和 (2.3) 式易见

$$
|\nabla B|^{2} \geqslant \frac{n+2|\nabla \sigma|^{2}}{4 n} \text {. }
$$

这蕴含着

$$
2(n-1) \frac{|\nabla B|^{2}}{\sigma}+\frac{1}{4}(n-1)(n-6)|\nabla \log \sigma|^{2} \geqslant \frac{n-1}{4 n}(n-2)^{2}|\nabla \log \sigma|^{2} .
$$

于是,结合 (2.7) 式和 (2.9) 式, 便得

$$
-\sigma^{2} \widetilde{R} \geqslant \sigma^{2}-n(n-1) a c \sigma-2(n-1)\left(2-\frac{1}{p}\right)|B|^{4}+2 n(n-1) c|B|^{3} .
$$

以下我们分两种情况讨论.

(I) $c \geqslant 0$.

把 (2.3) 式导人 (2.10) 式, 就有 


$$
\begin{aligned}
-\sigma^{2} \widetilde{R} \geqslant \sigma^{3} & -n(n-1) a c \sigma-2(n-1)\left(2-\frac{1}{p}\right) \sigma|B|^{2} \\
+ & 2 n(n-1) c\left[1+(n-1)\left(2-\frac{1}{p}\right)(a-1)\right]|B|^{2} .
\end{aligned}
$$

条件 (2.2) 式表明 (2.11) 式右边的最后一项非负, 因此, (2.11) 式与 (2.3) 式给出

$$
\begin{gathered}
-\widetilde{R} \geqslant 1-2(n-1)\left(2-\frac{1}{p}\right)+\frac{1}{\sigma} n(n-1) c[2(n-1) \\
\left.\times\left(2-\frac{1}{p}\right)(a-1)-a\right] .
\end{gathered}
$$

显然,由 (2.2) 式,上式右边最后一项也非负. 这样，(2.4）式就得证.

(II) $c<0$.

若命

$$
b=n(n-1)(a-1) c,
$$

则 (2.3) 式变成

将 (2.14) 式代入 (2.10) 式,我们有

$$
\sigma=|B|^{2}+b
$$

$$
-\sigma^{2} \widetilde{R} \geqslant\left[1-2(n-1)\left(2-\frac{1}{p}\right)\right] \sigma^{2}+(n-1) L(\sigma),
$$

其中

$$
L(\sigma) \equiv\left[\left(8-\frac{4}{p}-\frac{1}{n-1}\right) b+n c\right] \sigma-2 b\left[\left(2-\frac{1}{p}\right) b+n c\right]
$$

是 $\sigma$ 的线性函数, 且由 (2.14) 式,它的定义域是 $[b,+\infty)$.

我们要证明: 对 $\sigma \geqslant b$, 有 $L(\sigma) \geqslant 0$.

事实上,根据条件 $(2.2)_{2}$ 式,易见

$$
\begin{aligned}
L^{\prime}(\sigma) & \equiv\left(8-\frac{4}{p}-\frac{1}{n-1}\right) b+n c \\
& =\left\{\left[4(n-1)\left(2-\frac{1}{p}\right)-1\right](a-1)+1\right\} n c \geqslant 0 .
\end{aligned}
$$

因此,只要证明

$$
L(b) \equiv\left[2\left(2-\frac{1}{p}\right)-\frac{1}{n-1}\right] b^{2}-n c b \geqslant 0 .
$$

住意到 $c<0,(2.17)$ 式是 (2.13) 和 (2.2) 式的直接结果.

现在, 从 (2.15) 式和 $L(\sigma) \geqslant 0$, 即得 (2.4) 式.

定理 1 完全证毕.

因为对任何 $n \geqslant 2$ 和 $p \geqslant 1$, 易知

$$
1+1 /\left[2(n-1)\left(2-\frac{1}{p}\right)-1\right] \leqslant 2
$$

和

$$
1-1 /\left[4(n-1)\left(2-\frac{1}{p}\right)-1\right] \geqslant \frac{2}{3}
$$


所以, 从定理 1 立即推得下列

推论 1.1. 设 $F^{n+p}(c),\left(M^{n}, g\right)$ 及 $R$ 如定理 1 所述. 令 $\tilde{c}=\max \left\{c, \frac{1}{3} c\right\}$. 那末，共 形度量 $\tilde{g}=[2 n(n-1) \tilde{c}-R]$ 的纯量曲率 $\widetilde{R}$ 满足 $(2.4)$ 式.

由于 $n /(n-1) \geqslant 1+1 /\left[2(n-1)\left(2-\frac{1}{p}\right)-1\right]$, 故又有:

推论 1.2. 在定理 1 的同样假设下, 若 $c>0$, 则共形度量 $\tilde{g}=\left(n^{2} c-R\right) g$ 的纯量曲 率 $\widetilde{R}$ 满足 $(2.4)$ 式.

注. (2.4) 式是一个通用估计, 当 $c=0$ 和 $n=2$ 时, 上界可用 1 改进 ${ }^{[3]}$. 在 $n=2$ 和 $p=1$ 的情况, 当 $c>0$ 时取 $a=2$, 当 $c<0$ 时取 $a=0$,则从定理 1 便得 Barbosa-Do Carmo 的结果 ${ }^{[3]}$. 他们也得到了 $c=0$ 和 $p=1$ 的情况的类似估计 ${ }^{[1]}$.

\section{三、极小曲面的稳定性}

先考虑一般情况，设 $\left(M^{n}, g\right)(n \geqslant 2)$ 是单连通空间形式 $\boldsymbol{F}^{n+p}(c)$ 的极小子流形. 我 们采用类似于文献 [3] 的方法. 以后, $\mathscr{D} \subset M^{n}$ 总是指一个单连通区域，具有紧致闭包和逐 片光滑边界 $\partial \mathscr{D}$.

假定 $\mathscr{D} \subset M^{n}$ 不稳定. 根据 Smale 的 Morse 指标定理(见文献 [10] 或文献 [11])，存 在区域 $\mathscr{D}^{\prime} \subseteq \mathscr{D}$ 和 $\overline{\mathscr{D}}^{\prime}$ 上的 Jacobi 场 $V$, 使得 $V$ 在 $\partial \mathscr{D}^{\prime}$ 上消失. 由于单连通空间形式 $\boldsymbol{F}^{n+p}(c)$ 是实解析的, 故 $\boldsymbol{F}^{n+p}(c)$ 中的极小浸入也是实解析的. 从而, 作为椭圆方程组 (1.14) 的解，Jacobi 场 $V$ 也是实解析的. 所以,不失一般性, 可以假定 $V$ 在 $\mathscr{D}^{\prime}$ 中只有孤立 零点 $x_{1}, \cdots, x_{q}$. 在这些点之外, 我们可选取适当的标架场 $\left\{e_{A}\right\}$, 使得

$$
V=u e_{n+1} \text {, 在 } \mathscr{D}^{\prime} /\left\{x_{1}, \cdots, x_{q}\right\} \text { 上 } u>0 \text {, 且 }\left.u\right|_{a \mathscr{D}^{\prime}}=0 \text {. }
$$

关于这样的标架, (1.14) 式的 $e_{n+1}$ 方向的分量 $v_{n+1}$ 变成

$$
\Delta u+n c u+\left|B_{n+1}\right|^{2} u=0 \text {, }
$$

其中 $\left|B_{n+1}\right|^{2}=\sum_{i, 1}\left(h_{i n}^{n+1}\right)^{2}$. 因为

$$
\left|B_{n+1}\right|^{2}=|B|^{2}-\sum_{\beta \neq n+1}\left|B_{\beta}\right|^{2}=n(n-1) c-R-\sum_{\beta \neq n+1}\left|B_{\beta}\right|^{2},
$$

故从 (3.1) 式得, 在 $\mathscr{D}^{\prime} /\left\{x_{1}, \cdots, x_{q}\right\}$ 上

$$
u \Delta u+\left(n^{2} c-R\right) u^{2} \geqslant 0 .
$$

利用 Stokes 定理及 $\left.u\right|_{\partial \not \partial}=0$ ，从(3.2) 式易见

$$
\int_{\mathscr{D ^ { \prime }}}\left(n^{2} c-R\right) u^{2}{ }^{*} 1_{g} \geqslant \int_{\mathscr{D}^{\prime}}|\nabla u|^{2 *} 1_{g},
$$

这是我们下面要用到的基本不等式.

现在我们转到 $n=2$ 的情况,即 $F^{2+p}(c)$ 的极小曲面 $\left(M^{2}, g\right)$. 设 $K$ 是关于 $g$ 的 Gauss 曲率, 则 (3.3) 式变为:

$$
\int_{g} 2(2 c-K) u^{2 *} 1_{g} \geqslant \int_{g,}|\nabla u|^{2 *} 1_{g} .
$$

定理 2. 设 $\left(M^{2}, g\right)$ 是单连通空间形式 $\boldsymbol{F}^{2+p}(c)$ 的极小曲面. 令 


$$
\tilde{c}=\max \left\{c, \frac{3 p-2}{7 p-4} c\right\} .
$$

若 $\mathscr{D} \subset M^{2}$ 是单连通区域, 使得

$$
\int_{\Delta}(2 \tilde{c}-K){ }^{*} 1_{g}<2 \pi /\left(2-\frac{1}{p}\right),
$$

其中 $K$ 是 $\left(M^{2}, g\right)$ 的 Gauss 曲率, 则 $\mathscr{D}$ 是稳定的.

证. 采用反证法. 假定 $\mathscr{D}$ 不稳定, 则存在区域 $\mathscr{D}^{\prime} \subseteq \mathscr{D}$ ，使 (3.4) 式成立. 红此多 $\tilde{c}$ 的定义, 可得

$$
\int_{\mathscr{D}^{\prime}} 2(2 \tilde{c}-K) u^{2 *} 1_{g} \geqslant \int_{\mathscr{D}^{\prime}}|\nabla u|^{2 *} 1_{g} .
$$

在 $M^{2}$ 上引人共形度量 $\tilde{g}=2(2 \tilde{c}-K) g,(3.6)$ 式化为:

$$
\int_{\mathscr{D}^{\prime}} u^{2 *} 1_{\tilde{g}} \geqslant \int_{\mathscr{D}^{\prime}}|\nabla u|^{2 *} 1_{\tilde{g}},
$$

其中 ${ }^{*} 1_{\tilde{g}}$ 表示关于度量 $\tilde{g}$ 的 $M^{2}$ 的面积元. 利用极大一极小原理 ${ }^{[23]},(3.7)$ 式意味着

$$
1 \geqslant \frac{\int_{\mathscr{\mathscr { D } ^ { \prime }}}|\nabla u|^{2 *} 1_{\tilde{g}}}{\int_{\mathscr{D}} u^{2 *} 1_{\tilde{g}}} \geqslant \tilde{\lambda}_{1}\left(\mathscr{D}^{\prime}\right),
$$

其中 $\tilde{\lambda}_{1}\left(\mathscr{D}^{\prime}\right)$ 表示 $\overline{\mathscr{D}}^{\prime}$ 上关于 $\tilde{g}$ 的 Laplacian 的第一 Dirichlet 特征值.

另一方面,由定理 1 及其推论,关于 $\tilde{g}$ 的 Gauss 曲率 $\widetilde{K}$ 满足

$$
\widetilde{K} \leqslant \frac{1}{2}\left(3-\frac{2}{p}\right) \stackrel{(\mathrm{def})}{=} k_{p}
$$

根据文献 [3] 的命题 (3.3) 及推论 (3.20), 得

$$
\tilde{\lambda}_{1}\left(\mathscr{D}^{\prime}\right) \geqslant \lambda_{1}(Q) \text {, }
$$

其中 $\lambda_{1}(Q)$ 是曲率为 $k_{p}$ 的球面 $S^{2}\left(\frac{1}{\sqrt{k_{p}}}\right)$ 上测地盘 $\Omega$ 的 Laplacian 第一特征值, 这里 $Q$ 的 面积 $A(Q)=\tilde{A}\left(\mathscr{D}^{\prime}\right)$ ，后者表示 $\mathscr{D}^{\prime}$ 在度量 $\tilde{g}$ 下的面积. 但在假设 (3.5) 式下,有

$$
A(\Omega)=\int_{g}{ }^{*} 1_{\tilde{g}} \leqslant \int_{\infty} 2(2 \tilde{c}-K)^{*} 1_{g}<4 \pi /\left(2-\frac{1}{p}\right) .
$$

根据文献 [3] 中命题 (3.10) 的类似结论, 对于 $S^{2}\left(\frac{1}{\sqrt{k_{p}}}\right)$ 上的测地盘 $Q$, 可以估计 $\lambda_{1}(\Omega)$ 如下: 若 $A(Q)<2 \pi / k_{p}$, 则 $\lambda_{1}(Q)>2 k_{p}=3-\frac{2}{p} \geqslant 1$. 若 $2 \pi / k_{p} \leqslant A(Q)<4 \pi /(2-$ $\left.\frac{1}{p}\right)$, 则 $p \geqslant 2$, 并且 (3.10) 式蕴含 (参考文献 [3] 的命题 (3.10), (ii))

$$
\lambda_{1}(Q) \geqslant 2 k_{p}\left(\frac{4 \pi / k_{p}}{A(Q)}-1\right)=\frac{8 \pi}{A(Q)}-3+\frac{2}{p}>1 .
$$

因此, (3.8), (3.9) 式和 $\lambda_{1}(\theta)>1$ 就得出矛盾, 从而定理 2 得证.

注. 显然, 由文献 [3] 的定理 (1.4), 当 $c=0$ 和 $p \geqslant 3$ 时, 定理 2 的条件 (3.5) 式不是 最好. 当 $c>0$ 时, 这个结果已被 Do Carmo ${ }^{[3]}$ 宣布. 当 $c<0$ 时, 定理 2 改进了文献 [5] 的定理 4 . 
推论 2.1. 设 $\left(M^{2}, g\right)$ 是单连通的 4 维空间形式 $\boldsymbol{F}^{4}(c)$ 的极小曲面,令

$$
\tilde{c}=\max \left\{c, \frac{2}{5} c\right\} \text {. }
$$

若 $\mathscr{D} \subset M^{2}$ 是单连通区域,并且

$$
\int_{\mathscr{D}}(2 \tilde{c}-K)^{*} 1_{g}<\frac{4}{3} \pi,
$$

则 $\mathscr{D}$ 是稳定的.

\section{四、极小子流形的稳定性}

本节中我们总假定 $n \geqslant 3$, 并用 $\omega_{n}$ 表示单位 $n$ 维球面 $S^{n}(1) \hookrightarrow \mathbb{R}^{n+1}$ 的 $n$ 维体积, 即

$$
\omega_{n}=2 \pi^{(n+1) / 2} / \Gamma\left(\frac{n+1}{2}\right),
$$

这里 $\Gamma(t)$ 是 $\mathrm{Gamma}$ 函数.

定理 3. 设 $\left(M^{n}, g\right)$ 是单连通空间形式 $F^{n+p}(c)$ 的 $n(\geqslant 3)$ 维极小子流形. 用 $R$ 表 示关于 $g$ 的纯量曲率, 令

$$
\tilde{c}=\max \left\{n^{2} c, n(n-1)\left(1-1,\left[4(n-1)\left(2-\frac{1}{p}\right)-1\right]\right) c\right\} .
$$

设共形度量 $\tilde{g}=(\tilde{c}-R) g$ 具有常数截曲率. 若对于单连通区域 $\mathscr{D} \subset M^{n}$ 有

$$
\int_{\mathscr{D}}(\tilde{c}-R)^{*} 1_{s} \leqslant \frac{1}{2}(\sqrt{n})^{n}\left(4-\frac{2}{p}-\frac{1}{n-1}\right)^{-n / 2} \omega_{n},
$$

则 $\mathscr{T}$ 是稳定的.

证. 证明方法类似于定理 2. 我们从 (3.3) 式开始. 由 $\tilde{c}$ 的定义和 (3.3) 式得

$$
\int_{\mathscr{D}^{\prime}}(\tilde{c}-R) u^{a^{*}} 1_{g} \geqslant \int_{\text {边品 }}|\nabla u|^{2 *} 1_{g},
$$

利用共形度量 $\tilde{g}=(\tilde{c}-R) g$, 这就意味着

$$
1 \geqslant \bar{\lambda}_{1}\left(\mathscr{D}^{\prime}\right),
$$

其中 $\tilde{\lambda}_{1}\left(\mathscr{D}^{\prime}\right)$ 是 $\mathscr{D}^{\prime}$ 上关于 $\tilde{g}$ 的 Laplacian 的第一Dirichlet 特征值.

因为 $\tilde{g}$ 具有常数截曲率, 譬如 $\widetilde{K}$, 则关于 $\tilde{g}$ 的纯量曲率 $\widetilde{R}=n(n-1) \widetilde{K}$. 因此, 由定理 1 及推论 1.2 ,

$$
\widetilde{K} \leqslant \frac{1}{n}\left(4-\frac{2}{p}-\frac{1}{n-1}\right) \stackrel{(\mathrm{def})}{=} k .
$$

现把 $(\mathscr{D}, \tilde{g})$ 看作单连通空间形式 $\boldsymbol{F}^{n}(\widetilde{K})$ 的一个区域. 如所知, 在 $\boldsymbol{F}^{n}(\widetilde{K})$ 中，几何 等周不等式成立 (见文献 [14] 和文献 [15])，故 Faber-Krahn 不等式也成立（见 文献 [16]， p.87)。这样,由 Faber-Krahn 不等式得

$$
\tilde{\lambda}_{1}\left(\mathscr{D}^{\prime}\right) \geqslant \tilde{\lambda}_{1}(\Omega),
$$

其中 $Q$ 是 $F^{n}(\widetilde{K})$ 的一个测地盘, 使 $Q$ 的体积 $\widetilde{V}(Q)$ 等于 $\mathscr{D}^{\prime}$ 在度量 $\tilde{g}$ 下的体积 $\widetilde{V}\left(\mathscr{D}^{\prime}\right)$.

再根据郑绍远的特征值比较定理 ${ }^{[13]},(4.3)$ 式导致

$$
\bar{\lambda}_{1}(\Omega) \geqslant \lambda_{1}\left(\Omega^{*}\right),
$$

其中 $Q^{*}$ 是曲率为 $k$ 的 $n$ 维球面 $S^{n}\left(\frac{1}{\sqrt{k}}\right)$ 上的测地盘, 使 $\operatorname{Vol}\left(\Omega^{*}\right)=\widetilde{V}(\Omega)$. 但在条件 (4.1) 
式下,有

$$
\operatorname{Vol}\left(\Omega^{*}\right)=\widetilde{V}\left(\mathscr{D}^{\prime}\right) \leqslant \int_{\mathscr{D}}(\tilde{c}-R)^{*} 1_{g} \leqslant \frac{1}{2} \omega_{s}(\sqrt{k})^{-n},
$$

这表明 $Q^{*}$ 含在 $S^{n}\left(\frac{1}{\sqrt{k}}\right)$ 的闭半球面内, 后者的第一特征值等于 $n k$. 因此, 结合 (4.2), (4.4) 和 (4.5) 式, 可得

$$
1 \geqslant \lambda_{1}\left(Q^{*}\right) \geqslant n k=4-\frac{2}{p}-\frac{1}{n-1}>1,
$$

这是矛盾的. 定理 3 证毕.

定理 4. 设 $\left(M^{n}, g\right)$ 是单连通空间形式 $F^{n+p}(c)(c \geqslant 0)$ 的 $n(\geqslant 3)$ 维极小子流形, 它的第二基本形式 $B$ 具有常数长度 $2 b=|B|^{2}$. 若 $\mathscr{D} \subset M^{n}$ 是测地盘, 使得

$$
\operatorname{Vol}(\mathscr{D})<\frac{1}{2} \omega_{n}(2 b+n c)^{(n-2) / 2}(b+c)^{-n / 2},
$$

则 $\mathscr{D}$ 是稳定的.

证. 因为 $|B|^{2}=2 b$, 从 (1.6) 式则得

$$
n^{2} c-R=2 b+n c \text {. }
$$

这样, (4.7) 式可改写为:

$$
\int_{\mathscr{D}}(2 b+n c)^{*} 1_{g}<\frac{1}{2} \omega_{n} \cdot\left(\frac{b+c}{2 b+n c}\right)^{-n / 2} \text {. }
$$

如同定理 3 的证明所见, 对于 $\mathscr{D}$ 的稳定性, 只要证明相似度量 $\tilde{g}=(2 b+n c) g$ 的截曲 率 $\widetilde{K}_{M}$ 满足

$$
\widetilde{K}_{M} \leqslant \frac{b+c}{2 b+n c}
$$

但在相似变换 $\tilde{g}=(26+n c) g$ 下, 有 ${ }^{[12]}$

$$
K_{M}=(2 b+n c) \widetilde{K}_{M},
$$

其中 $K_{M}$ 是关于 $g$ 的截曲率. 所以只要证明 $K_{M} \leqslant b+c$.

为此, 回到 Gauss 方程 (1.4). 显然, 对于任何 $i \neq j$, 我们有

$$
\begin{aligned}
R_{i j i j} & =c+\sum_{a} h_{i j}^{a} h_{i j}^{a}-\sum_{a}\left(h_{i j}^{a}\right)^{2} \\
& \leqslant c+\frac{1}{2} \sum_{a}\left[\left(h_{i i}^{a}\right)^{2}+\left(h_{j i}^{a}\right)^{2}\right] \leqslant c+\frac{1}{2}|B|^{2},
\end{aligned}
$$

此式连同 $|B|^{2}=2 b$, 便得

这就是所要证的. 因此,定理 4 证毕.

$$
R_{i j i} \leqslant b+c \quad(i \neq j),
$$

作为特例 ${ }^{[8]}$, 我们有下列推论.

推论 4.1. 设 $\left(M^{n}, g\right)$ 是 $S^{n+1}(1)$ 的 Clifford 极小超曲面. 若 $\mathscr{D} \subset M^{n}$ 是测地盘, 使 得

则 $\mathscr{D}$ 是稳定的.

$$
\operatorname{Vol}(\mathscr{D})<2^{(n-2)} n^{(n-2) / 2}(n+2)^{-n / 2} \cdot \omega_{n},
$$


推论 4.2. 设 $S^{n}\left(\sqrt{\frac{2(n+1)}{n}}\right)$ 是 $S^{n+p}(1)$ 的 Veronese 极小子流形, 其中 $p=\frac{1}{2}(n-1)$ $(n+2)^{[83}$. 若 $\mathscr{D} \subset S^{n}\left(\sqrt{\frac{2(n+1)}{n}}\right)$ 是测地盘, 使得

$$
\operatorname{Vol}(\mathscr{D})<\frac{n+1}{n^{2}(n+3)}\left[\frac{n^{3}+(n+1)^{2}+3}{2 n^{2}(n+3)}\right]^{-n / 3} \cdot \omega_{n},
$$

则 $\mathscr{D}$ 是稳定的.

注. 众所㘯, $S^{n+p}(1)$ 中不存在紧致的稳定极小子流形 ${ }^{[10]}$. 定理 4 及其推论给出了这种 极小子流跎上稳定测地盘的体积大小。

\section{参 考 文 献}

[1] Barbosa, J. L. \& Do Carmo, M., An. Acad. Brasil. Ci., 50(1978), $451-454$.

[2] Chern, S. S. \& Osserman, R.. Remarks on the Riemanniun Mletric of a mmimul submanijold, Geom. Symp. Utrecht 1980. Springer Notes 894, 49-90.

[3] Barbosa, J. L. \& Do Carmo, M., Math, Z., 173(1980), 13-28.

[4] Burbosa, J. L. \& Do Carmo, M., Amer. J. Marh., 98(1976), 515--528

[5] Do Carmo, M., Stabiluy of minimal submanifolds, Springer, Notes 838, 129-139.

[6] Mut i. H., Yokahoma Math. J., 25(1977), 9-15.

[7] Siruck, K., Math. Z., 144(1975), 169-174.

[8] Chern, S. S., Do Carno. M. \& Kobayashi, S. Minimal Submanifolds of a sphere unith second fundamcntal form of constant length, Shiing-shen Chern Selected papers, Springur-Verlag, 1978, 393-409.

[9|Chern, S. S. Minimal subnanifolds in a Riemannian manifold, Mime. Lect. Notes, Univ. of Kansas, 1968.

[111) Simons, J., Ann. of Math, 88(1968), 62-105.

111] Snule, S., J. Math. Meih., 14(1965), 1049-1056.

[12] Chrn, B. Y., Geometry of submonifolds, Marcel Dekke: Inc., 197 ?

$113 j$ Chng. S. Y., Proc. Symp. Pure Math. A. M. S., 27(1975), Part 11, 185-193.

[14] Osserman, R., Bull. A. M. S, \$4(1978), 1182-1238.

[15] Payne, L. E., SHAM Rev', 9(1967), 453-488.

[16] Chavel, I., Proc. Symp. Pure Math. A. M. S., 36(1980), 79-89. 